\title{
Interpretation of Partial Discharges at dc Voltages
}

\author{
U. Fromm \\ High Voltage Laboratory, \\ Delft University of Technology, Delft, the Netherlands
}

\begin{abstract}
The aim of this paper is to give an overview on partial discharges at dc voltage. A model is presented which describes the stochastic discharge process. The model is experimentally verified for internal discharges and for corona in air. In addition, the classification of discharge patterns is discussed. The 3-dimensional histogram describing the number of discharges depending on the discharge magnitude and on the time to the successive discharge is suggested to be used as a base for discharge recognition at dc voltage. Experimental proof is presented to show the strength of this approach.
\end{abstract}

\section{INTRODUCTION}

$\mathrm{T}$ HE destructive character of partial discharges (PD) is well known. There is much knowledge about how to detect and evaluate characteristic discharge parameters at ac voltage. There is little published work on PD measurement at dc voltage. The majority of the measurements presented in the literature were carried out on simple sample configurations [1-7]. A few researchers report measurements on real industrial objects [8-12]. There is, however, a lack of knowledge in interpreting the measured data.

The stochastic properties of PD phenomena were reviewed by Van Brunt [13]. He discussed the basic physical mechanisms of discharge initiation and growth, including the stochastic behavior of corona in gases, and discharges that occur in liquid media and in the presence of solid dielectric interfaces.

PD in solid insulation (internal discharges) develop within voids or cracks that contain gas, or along the surfaces at gas-solid interfaces. One of the first studies on dc internal PD is reported by Roger and Skipper [14]. They calculated the discharge repetition rate assuming the residual voltage across the discharging void $V_{r}$ after discharge occurrence to be negligible compared to the ignition voltage $V_{i}$ of a discharge. If the residual voltage becomes larger, the discharge frequency increases. The dissipated energy increases as well but does not exceed twice the energy dissipated if $V_{r}=0$ [15]. Shihab [5] proved experimentally that there is a direct relation between the discharge frequency and the conductivity of the dielectric material. The study of the physical discharge mechanism in voids led to the introduction of the term 'Townsend-like' discharge and 'streamer-like' discharge by Devins [1]. Based on experimental and theoretical investigations, Fromm and Morshuis [16] concluded that the discharge mechanism in small voids is mainly Townsend-like.

In the following, the measurement of PD is discussed. A model is presented which describes the stochastic discharge process. The model is verified experimentally for internal discharges and for corona in air. The classification of discharge patterns is discussed in the last Section.

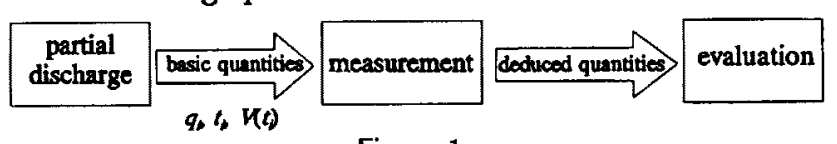

Figure 1.

Flowchart for the conventional discharge detection.

\section{MEASUREMENT OF dc PD}

PD are usually detected by integrating the displacement current in the leads of the test object. The measured value is the discharge magnitude, expressed in units of $\mathrm{pC}$.

Three basic quantities (see Figure 1) can be measured: the discharge magnitude $q_{i}$, the time of discharge occurrence $t_{i}$, and the instantaneous voltage across the test 
Table 1. List of symbols.

\begin{tabular}{|r|l|}
\hline$E_{\min }$ & minimal breakdown field strength \\
$\Delta E$ & excess field at discharge ignition \\
$K_{u}$ & kurtosis \\
$S_{k}$ & skewness \\
$V_{i}$ & ignition voltage across a void \\
$V_{r}$ & residual voltage across a void \\
$q$ & discharge magnitude \\
$\varphi$ & phase angle of the discharge event \\
$q_{i}$ & discharge magnitude of the $i$-th discharge \\
$t_{i}$ & time of occurrence of the $i$-th discharge \\
$\varphi_{i}$ & phase angle of the $i$-th discharge event \\
$t_{L}$ & time lag \\
$\bar{t}_{L}$ & mean time lag \\
$t_{R}$ & recovery time \\
$\bar{t}_{R}$ & mean recovery time \\
$t_{L, i}$ & the time lag of the $i$-th discharge \\
$t_{R, i}$ & the recovery time caused by the $i$-th discharge \\
$\Delta t$ & time separation between consecutive discharges \\
$\Delta t_{p r e}$ & time to the previous discharge \\
$\overline{\Delta t}_{\text {pre }}$ & mean time to the previous discharge \\
$\Delta t_{s u c}$ & time to the successive discharge \\
$\overline{\Delta t}_{\text {suc }}$ & mean time to the successive discharge \\
$\Delta t_{p r e(i)}$ & time between the $i$-th discharge and its predecessor \\
$\Delta t_{s u c(i)}$ & time between the $i$-th discharge and its successor \\
$H(q, \varphi)$ & number of discharges depending on $q$ and $\varphi$ \\
$H\left(q, \Delta t_{s u c}\right)$ & number of discharges depending on $q$ and $\Delta t_{s u c}$ \\
$H(q)$ & number of discharges depending on $q$ \\
$H\left(\Delta t_{s u c}\right)$ & number of discharges depending on $\Delta t_{\text {suc }}$ \\
\hline \hline
\end{tabular}

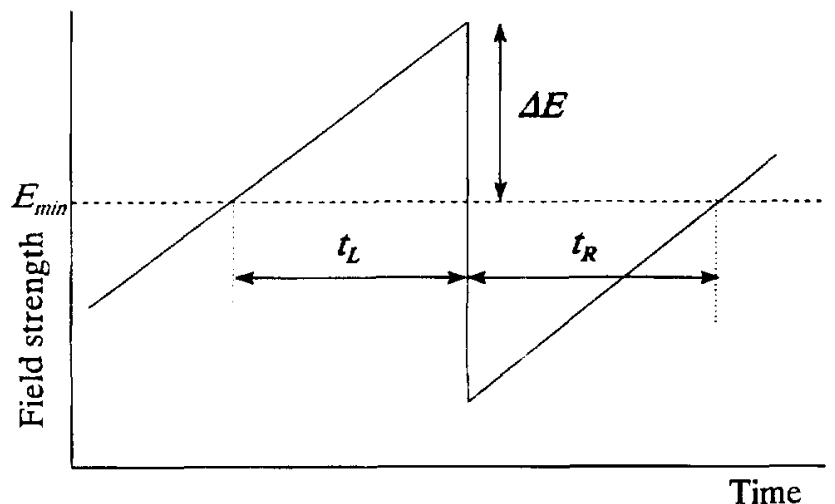

Figure 2.

The electric field strength at the discharge location.

object $V\left(t_{i}\right)$. These basic quantities are related to the occurrence of a single discharge.

If the shape of the voltage applied to the test object is known, the number of basic quantities can be reduced. At ac the voltage is given by $V\left(t_{i}\right)=V \sin \left(\omega t_{i}\right)=$ $V \sin \left(\varphi_{i}\right)$. Usually the phase angle $\varphi_{i}$ is recorded instead of $t_{i}$ and $V\left(t_{i}\right)$. At dc the voltage is constant and the number of basic quantities reduces to the discharge magnitude $q_{i}$, and the time of discharge occurrence $t_{i}$.

The discharge phenomena have a stochastic nature, so

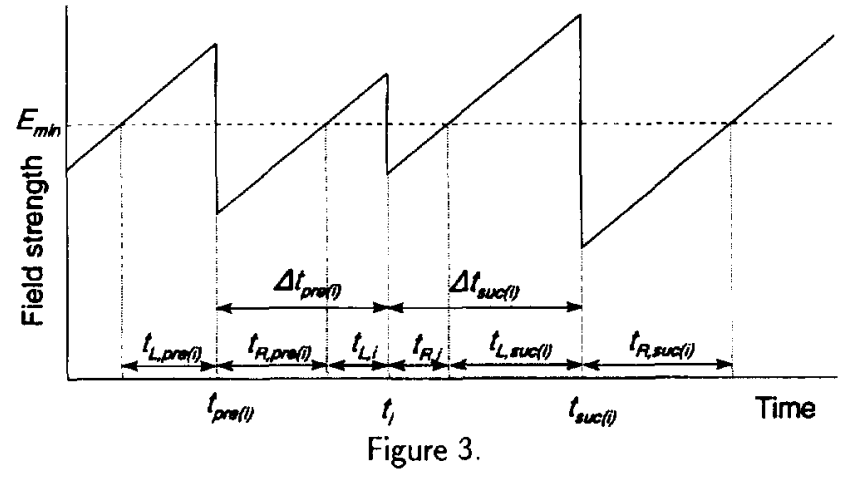

The relationship between the time lag $t_{L}$, the recovery time $t_{R}$ and the time to the previous and successive discharge.

that during the measurement a large set of basic quantities can be recorded. One of the problems of measuring PD is to reduce the large amount of basic quantities to a few deduced quantities, sufficient for an evaluation (Figure 1). At ac voltage most systems today measure the $H(q, \varphi)$ histogram (number of discharges depending on the discharge magnitude $q$ and on the phase angle $\varphi$ ) or one of its derivatives. For dc voltage there is no generally accepted standard yet. In the literature different data displays are used. Among these are the time 
functions of the discharge frequency [5] and the apparent discharge current [17], the distribution functions for the discharge magnitude $[4,5,18]$, time $[2,19]$, conditioned distributions [20], or the 3-dimensional $H\left(q, \Delta t_{\text {suc }}\right)$ distribution $[21,22]$.

Examples of other displays are the discharge frequency as a function of the discharge magnitude $[4,9,22]$ and the discharge signal as recorded on a strip chard recorder [23].

In order to study all these possible deduced quantities, a measurement system as described in [2] was used. The basic quantities discharge magnitude $q_{i}$ and the time of discharge occurrence $t_{i}$ were recorded in the memory of a personal computer. Specially developed software enables the on-line display of different deduced quantities.

\section{THE TIME LAG/RECOVERY MODEL}

In order to cause a discharge, two conditions must be fulfilled.

The electric field at the location where the discharge starts, must exceed the minimal breakdown field strength $E_{\min }$ (Figure 2), and a starting electron must be present. Waiting for this electron results in a time lag $t_{L}$ of the discharge ignition. During $t_{L}$ the field increases to a value above the minimal field, so that the discharge ignites at an excess field $\Delta E$ (Figure 2). The field increase in Figures 2 and 3 is simplified to be linear, whereas it must be assumed to be nonlinear in general. However, for the proposed model the time dependence of the field increase is not important.

The occurrence of PD causes accumulation of charge near the discharge location. This leads to a drop of the local field strength below the minimal breakdown field strength $E_{\min }$, so that the discharge extinguishes. In order to reach again the minimal breakdown field the recovery time $t_{R}$ is required (Figure 2). The model is based on the assumption that time lag $t_{L}$, the discharge magnitude $q$ and the recovery time $t_{R}$ are mutually related.

$$
\begin{aligned}
& q=f_{1}\left(t_{L}\right) \\
& t_{L}=f_{1}^{*}(q) \\
& t_{R}=f_{2}(q)
\end{aligned}
$$

In order to make statistical considerations the following symbols are used. The discharge occurring at $t_{i}$ has a time lag $t_{L, i}$ and affects the recovery time $t_{R, i}$ (Figure 3 ). The predecessor of the discharge at $t_{i}$ occurred at $t_{p r e(i)}$, the successor occurs at $t_{s u c(i)}$. The time between the discharge at $t_{i}$ and its predecessor is $\Delta t_{\text {pre(i) }}$. The time between the discharge at $t_{i}$ and its successor is $\Delta t_{s u c(i)}$.

Further it is assumed that the discharge magnitude does not depend on the previous discharge as will be verified in the experimental Section. The independence means that a discharge does not 'remember' the magnitude of the previous discharge and does not influence the magnitude of the successive discharge. Therefore the mean time lag of the successors of $n$ arbitrarily chosen elements of a set of discharges is equal to the mean time lag of all discharges.

$$
\frac{1}{n} \sum_{j=1}^{n} t_{L, s u c(i)}=\overline{t_{L}}
$$

Similarly, the mean recovery time of the predecessor of $n$ arbitrarily chosen elements of a set of discharges is equal to the mean recovery time of all discharges.

$$
\frac{1}{n} \sum_{j=1}^{n} t_{R, p r e(i)}=\overline{t_{R}}
$$

The time to the previous discharge is the sum of the time lag (which determines the discharge magnitude) and the recovery time of the previous discharge (Figure 3 ).

$$
\Delta t_{\text {pre }(i)}=t_{R, p r e(i)}+t_{L, i}
$$

Similarly, the time to the successive discharge is the sum of the recovery time and the time lag of the successive discharge.

$$
\Delta t_{\text {suc }(i)}=t_{R, i}+t_{L, s u c(i)}
$$

Let us consider $n$ discharges. From Equation (5) the mean time to the previous discharge is

$$
\overline{\Delta t}_{p r e}=\frac{1}{n} \sum_{j=1}^{n}\left(t_{R, p r e(i)}+t_{L, i}\right)
$$

Let us further assume that all $n$ (not necessary consecutive) discharges are of the same discharge magnitude $q_{i}=q$. Then Equation (1) leads to $t_{L, i}=t_{L}(q)$, so that Equation (7) can be rewritten as

$$
\overline{\Delta t}_{\text {pre }}(q)=t_{L}(q)+\frac{1}{n} \sum_{j=1}^{n}\left(t_{R, p r e(i)}\right.
$$

As mentioned above, the successive discharges are not correlated, so that the predecessors (with the recovery time $\left.t_{R, p r e(i)}\right)$ of the discharges of the magnitude $q_{i}$ can be assumed as arbitrary chosen. Then Equation (4) and (8) lead to

$$
\overline{\Delta t}_{p r e}(q)=t_{L}(q)+\bar{t}_{R}
$$

The time to the successor is, analogously,

$$
\overline{\Delta t}_{s u c}(q)=t_{R}(q)+\overline{t_{L}}
$$

Consequently, the model predicts a mean time interval between discharges that is different for previous and successive discharges for a fixed magnitude $q$. 

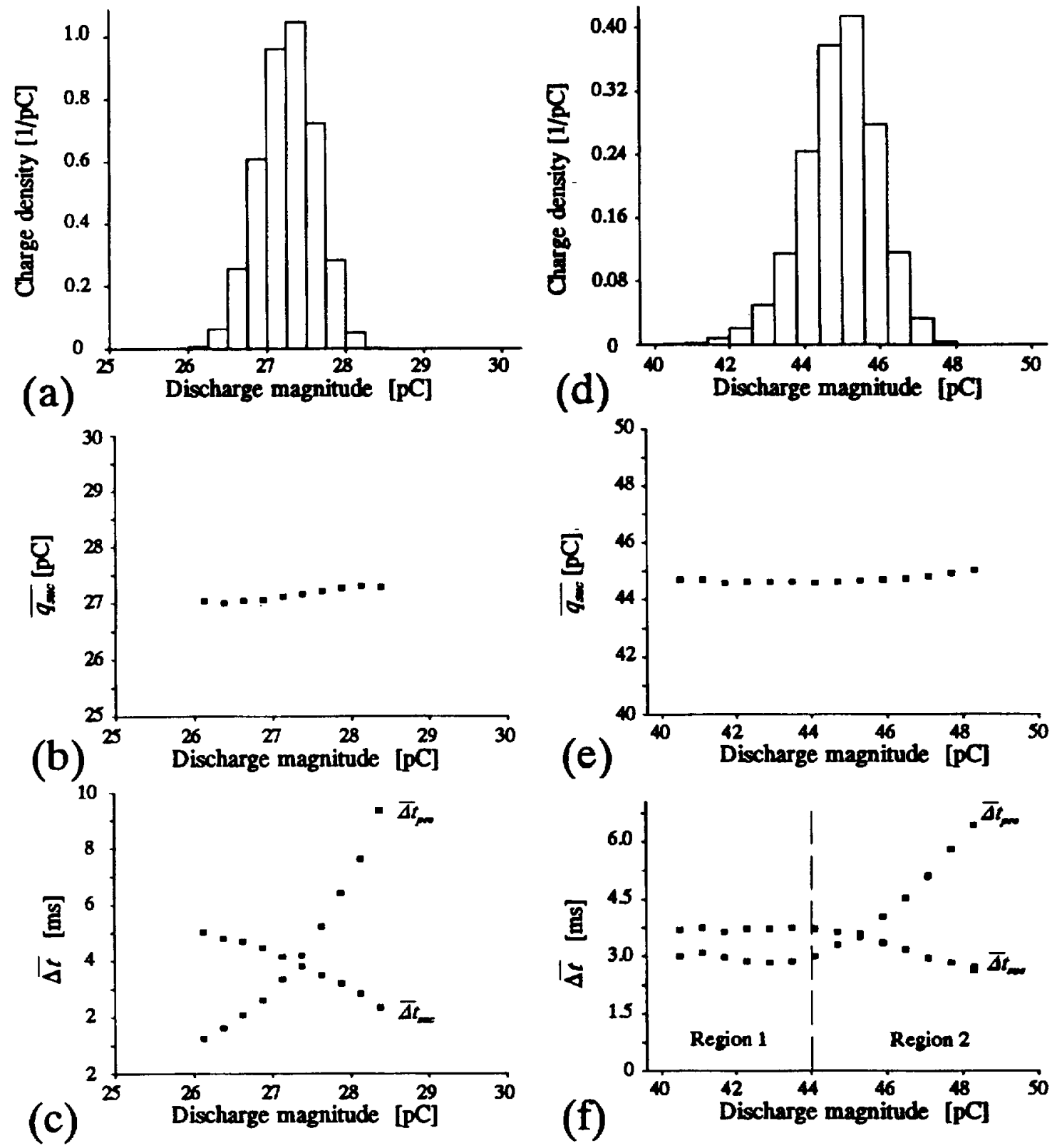

Figure 4.

Results for the discharge measurements. (a), (b), (c) negative needle at $V_{0}=6.5 \mathrm{kV}$; (d), (e), (f) positive needle at $V_{0}=8 \mathrm{kV}$. Discharge magnitude histogram: (a), (d). Mean discharge magnitude as function of the discharge magnitude: (b), (e) Mean time to the previous discharge $\overline{\Delta t}_{\text {pre }}$ and mean time to the successive discharge $\overline{\Delta t}_{\text {suc }}$ as function of the discharge magnitude: (c), (f).

In order to verify the model, the measured discharge data set is divided into subsets with a constant discharge magnitude. The mean time to the successor and to the predecessor can be evaluated for each discharge magnitude. The calculated values are plotted in a $q-\overline{\Delta t}_{\text {suc }}$ diagram and a $q-\overline{\Delta t}_{p r e}$ diagram. If the two diagrams show a continuous relation between the discharge magnitude and the mean time to the successor, and a continuous relation between discharge magnitude and the mean time to the predecessor, the model as developed above is realistic.

\section{EXPERIMENTAL}

\subsection{CORONA DISCHARGES}

Corona discharges were generated in front of a needle electrode. The distance between the HV needle and the ground electrode is very large $(>0.5 \mathrm{~m})$ compared to the needle radius $(\approx 40 \mu \mathrm{m})$. The statistical behavior 
is discussed for negative corona measured at $6.5 \mathrm{kV}$ and for positive corona measured at $8 \mathrm{kV}$, which is 15 to $20 \%$ above inception voltage. The distributions of the discharge magnitude for negative and positive corona are shown in Figures 4(a) and (d) respectively.

The average magnitude of the successive discharge as a function of the discharge magnitude for negative and positive corona is shown in Figures 4 (b) and (e) respectively. The average magnitude of the successive discharge does not depend on the discharge magnitude which supports one of the assumptions of the time lag/recovery model. The average time to the predecessor $\overline{\Delta t}_{\text {pre }}$ and to the successor $\overline{\Delta t}_{s u c}$ as a function of the discharge magnitude are shown for negative corona in Figure 4(c) and for positive corona in Figure 4(f).

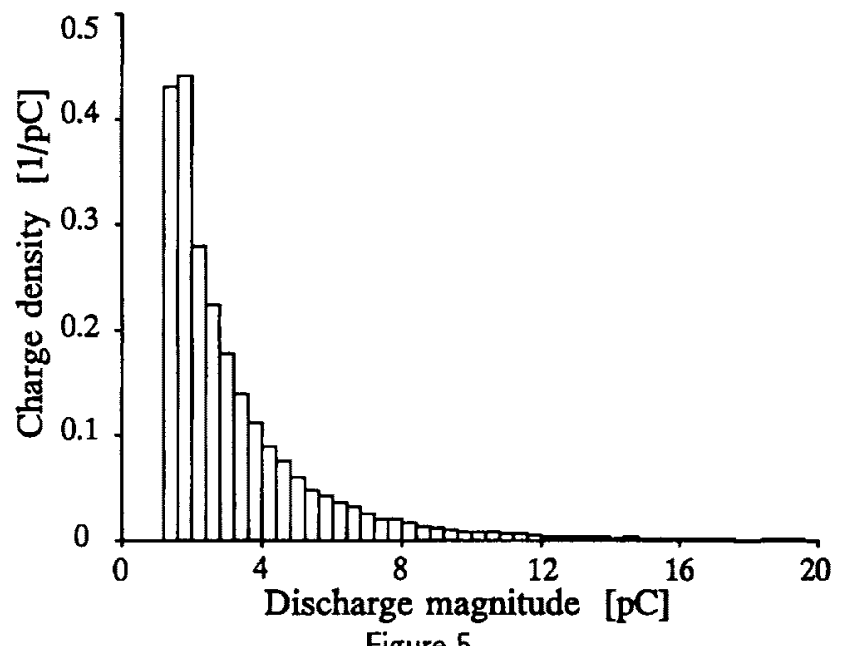

Figure 5.

The density function of the discharge magnitude (internal discharges, test voltage $20 \mathrm{kV}$, sensitivity $1.5 \mathrm{pC})$.

\subsubsection{NEGATIVE CORONA}

Equation (9) and Figure 4(c) lead to the conclusion that an enlarged discharge magnitude is caused by an prolonged time lag. Such behavior is expected, because a prolonged time lag leads to a discharge ignition at higher field strength which in its turn causes a larger discharge magnitude, because more space charge is required to extinguish the discharge process.

Equation (10) and Figure 4(c) lead to the conclusion that an enlarged discharge magnitude causes a shorter recovery time. Such a behavior can be explained as follows. The dependence of the recovery time $t_{R}$ on $q$ is determined by two competing effects.

First, the field reduction as a result of ion space charge generated by the previous discharge. In course of time the space charge disappears, again causing an increase of the field strength. A larger discharge magnitude is accompanied by a larger space charge, so that the recovery time $t_{R}$ (necessary to reach again the minimal breakdown field strength $E_{\min }$ ) will increase.

Second, the ionization coefficient is enhanced in the gas volume where the next discharge develops, caused by the metastable species generated by the previous discharge [13]. The enhanced ionization coefficient causes a decrease of the minimum breakdown field strength, so that a smaller recovery time is required in order to reach again the minimum breakdown field strength.

The second effect obviously dominates the measured data and leads to a decreased recovery time $t_{R}$ for an increased discharge magnitude. This behavior is in agreement with the results reported by Van Brunt [13].

\subsubsection{POSITIVE CORONA}

For positive corona the picture is not as clear as for negative corona. Two regions can be distinguished: Region 1 for discharges $\lesssim 44 \mathrm{pC}$ and region 2 for discharges $>44 \mathrm{pC}$ (see Figure $4(\mathrm{f})$ ).

In region 2 the mean time to the previous discharge $\overline{\Delta t}_{\text {pre }}$ increases for an increased magnitude whereas the mean time to the successive discharge $\overline{\Delta t}_{s u c}$ decreases. The explanation might be similar to that for negative corona. The dependence of the recovery time $t_{R}$ on $q$ is determined by the two competing effects (field reduction, and enhancement of the ionization coefficient). The second effect obviously dominates within region 2 and leads to the decreased $\overline{\Delta t}_{s u e}$ for an increased discharge magnitude.

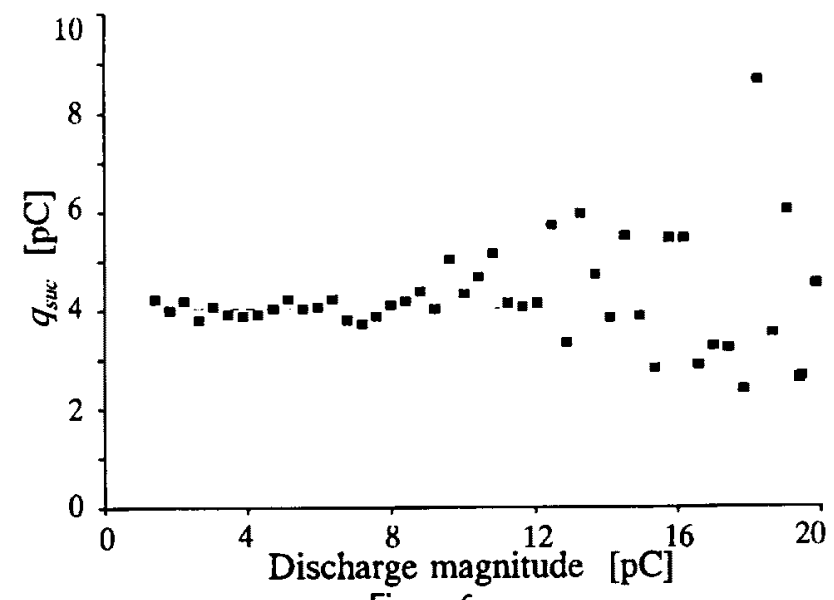

Figure 6.

The mean magnitude of the successor as a function of the discharge magnitude (internal discharges, test voltage $20 \mathrm{kV}$, sensitivity $1.5 \mathrm{pC}$ ).

In region 1 the time to the previous discharge $\overline{\Delta t}_{\text {pre }}$ and the mean time to the successive discharge $\overline{\Delta t}_{\text {suc }}$ are 
no function of the discharge magnitude (Figure $4(f)$ ). Together with the Equation (9) and (10) two conclusions are drawn.

First, the recovery time $t_{R}$ is no function of the discharge magnitude $q$. Such behavior can be explained by the compensation of the same two competing effects (as in negative corona).

Second, the time lag $t_{L}$ does not determine the discharge magnitude $q$. In order explain this, more investigations are required.

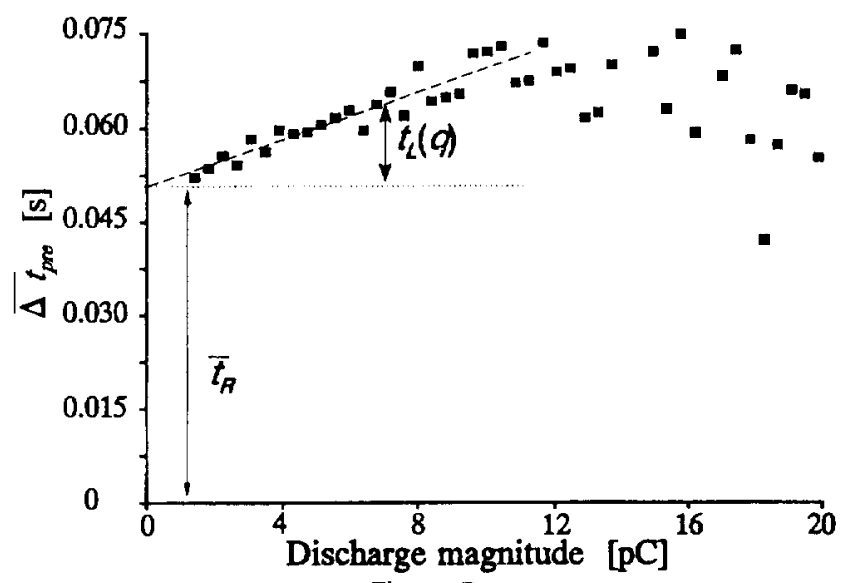

Figure 7

The time to the previous discharge as a function of the discharge magnitude (internal discharges, test voltage $20 \mathrm{kV}$, sensitivity $1.5 \mathrm{pC}$ ).

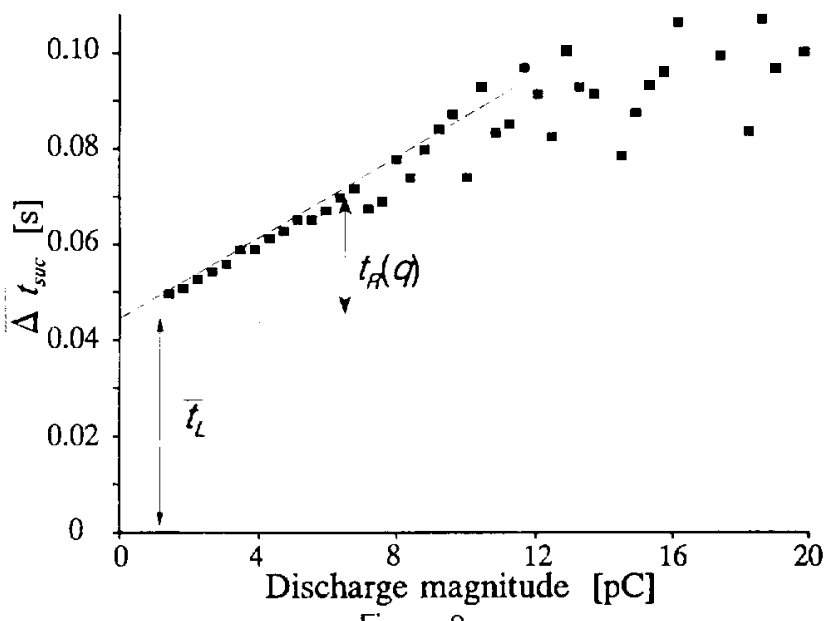

Figure 8

The time to the successive discharge as a function of the discharge magnitude (internal discharges, test voltage $20 \mathrm{kV}$, sensitivity $1.5 \mathrm{pC}$ ).

\subsection{INTERNAL PD}

As reported in $[22,24]$ the proposed time lag/recovery model can be used for the description of internal PD as well, if the whole void is discharging. This is almost true for Townsend-like discharges [25]. The measurements were made on sandwich-type samples ( 3 polyethylene foils, each $0.1 \mathrm{~mm}$ thick; the middle foil has a 6 mm diameter hole).

The measured density function of the discharge magnitude at $20 \mathrm{kV}$ is shown in Figure 5. In agreement with the results of other workers, $[1,5]$ the density is maximal for the smallest measured discharges.

The average magnitude of the successive discharge as a function of the discharge magnitude is shown in Figure 6. The average magnitude of the successive discharge does not depend on the discharge magnitude which supports one of the assumptions, the time lag/recovery model is based on.

The mean times to the predecessor and successor at different discharge magnitudes are shown in Figures 7 and 8, respectively. If one assumes that $t_{L} \rightarrow 0$ and $t_{R} \rightarrow 0$ for $q \rightarrow 0$ then with the Equation (9) and (10) the mean time lag and the mean recovery time can be determined by extrapolation

$$
\begin{aligned}
& \overline{\Delta t}_{\text {pre }}(q=0)=\overline{t_{R}} \\
& \overline{\Delta t}_{\text {suc }}(q=0)=\overline{t_{L}}
\end{aligned}
$$

For small discharge magnitudes, there is an almost linear relationship between $q, \overline{\Delta t}_{s u c}(q)$ and $\overline{\Delta t}_{p r e}(q)$. Using linear extrapolation, the values $\overline{\Delta t}_{s u c}(q=0)$ and $\overline{\Delta t}_{\text {pre }}(q=0)$ can be determined (Figures 7 and 8 ). The mean time lag and the mean recovery time are obtained to be $\overline{t_{R}} \approx 50 \mathrm{~ms}$, and $\overline{t_{L}} \approx 45 \mathrm{~ms}$. Further the relations $t_{L}=f_{1}(q)$ and $t_{L}=f_{2}(q)[\mathrm{ms} / \mathrm{pC}]$ can be reconstructed to be (see Figures 7 and 8 )

$$
\begin{aligned}
t_{L} & =f_{1}(q) \approx 2 q \\
q & =f_{1}^{*}\left(t_{L}\right) \approx 0.5 t_{L} \\
t_{R} & =f_{2}(q) \approx 4 q
\end{aligned}
$$

For large discharge magnitudes in Figures 6, 7 and 8, a rather large scatter is observed. The reasons therefore might be as follows.

1. The small density for large discharge magnitude leads to a small number of events per charge interval (Figure 5). The calculation of a mean value of a few events only leads to a larger scatter.

2. Time-resolved measurements have shown that streamer-like discharges have a larger magnitude than Townsend-like discharges $[1,24]$. The model is based on discharging of the complete void surface, which is approximately true for Townsend-like discharges, but not for streamer-like ones [25]. 


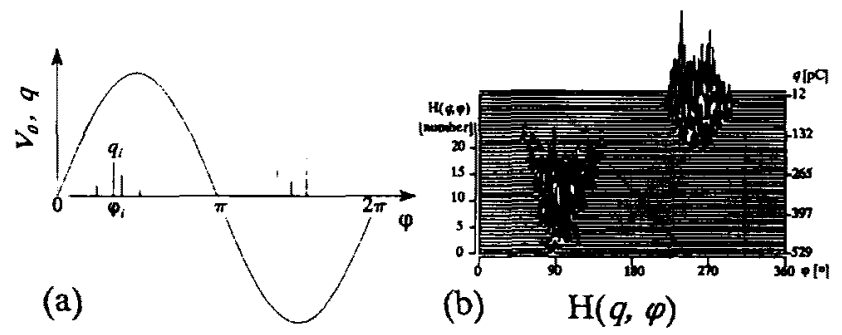

Figure 9.

The ac discharge process: (a) time function with discharge magnitude $q_{i}$ and phase angle $\varphi$ as basic quantities; (b) the $H(q, \varphi)$ distribution as characteristic discharge function (corona in oil measured with a conventional detector [37]).

The measured discharge repetition is much larger than that reported for practical test objects [8]. The residual voltage after discharge occurrence for Townsend-like discharges is very close to the ignition voltage [25]. This causes a small recovery time leading to the measured large discharge repetition. In the reported practical cases the low measured repetition rate may have been caused by the fact that the Townsend-like discharges (which have a very small magnitude) have not been registered or that only streamer-like discharges did occur.

\section{CLASSIFICATION OF dc PD}

The time lag/recovery model was developed to describe the discharge behavior of defects with a single discharge site. It can be used to study discharge properties based on statistical measurements. However, for practical classification purposes it should not be used, because its applicability is restricted to a few special cases.

For the classification of PD a more general approach is made. The main principle of discharge diagnostics is the following. PD occurring in the test object is measured and a 'finger print' can be derived for the defect generating the discharges. By comparing the finger print of an unknown defect with the finger prints of known defects, the unknown defect may be recognized.

\section{1. ac DISCHARGE DIAGNOSTICS}

The recognition and classification of PD has first been developed at ac voltage [26]. For each single discharge the discharge magnitude $q$ and the phase angle $\varphi$ can be measured. These quantities are named 'basic quantities'. As has been mentioned above, a finger print for the discharging defect is derived out of the measured basic quantities. In the beginning the discharge pulses where displayed superimposed on the cycle of the test voltage. The optical image was used as finger print [26]. An expert was required to relate the finger prints (characteristic discharge patterns) to certain types of defects. The availability of computers and the development of classification procedures made automated discharge classification and recognition possible. Therefore characteristic parameters are calculated from the measured data. These characteristic parameters, the finger print, are stored in a data bank. By comparing the characteristic parameters of an unknown defect with the parameters in the data bank the recognition of the unknown defect may be possible. For ac the following approaches are known to the author.

One group of classification procedures is based on 2dimensional histograms, for instance the phase angle histogram or the discharge magnitude histogram. Characteristic parameters for instance skewness and kurtosis are used [27-29] to describe the shape of the measured distributions. These characteristic parameters can be stored in a data base. The classification is made by comparing the measured characteristic parameters with those stored in the data base. In order to compare the characteristic parameters, different mathematical procedures, for instance neural networks $[28,29]$ or the centour score method $[30,31]$ are reported.

Another group of classification procedures is based on the 3-dimensional discharge magnitude-phase histogram $H(q, \varphi)$. Characteristic parameters are used to describe the histogram $H(q, \varphi)$. For instance the surface of the $H(q, \varphi)$ histogram was described with fractal features such as fractal dimension and lacunarity [32]. The fractal dimension can be used as a measure of the surface roughness and the lacunarity is used as a measure of the denseness of a fractal surface $[33,34]$. These fractal features were further used as characteristic parameters. Another approach is reported in $[35,36]$, where the 3 -dimensional discharge magnitude-phase histogram $H(q, \varphi)$ is directly used as a set of characteristic parameter. The classification is made by neural networks $[35,36]$.

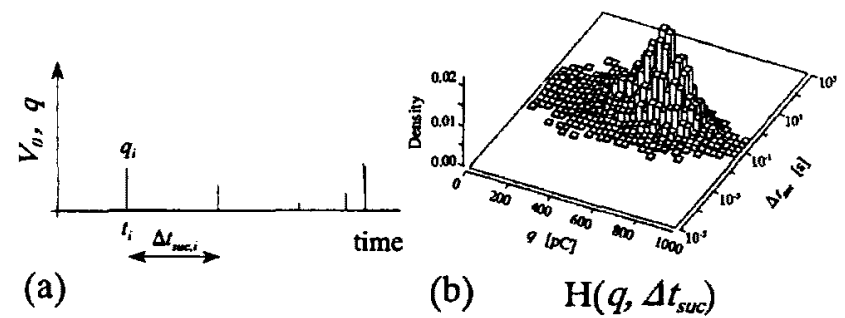

Figure 10

The dc discharge process: (a) time function with discharge magnitude $q_{i}$ and time of occurrence $t_{i}$ as basic quantities; (b) the $H\left(q, \Delta t_{s u c}\right)$ distribution as characteristic discharge function (corona in oil).

All the 2-dimensional distributions used in the first group can be derived from the 3-dimensional discharge magnitude-phase histogram $H(q, \varphi)$. Therefore it can be 


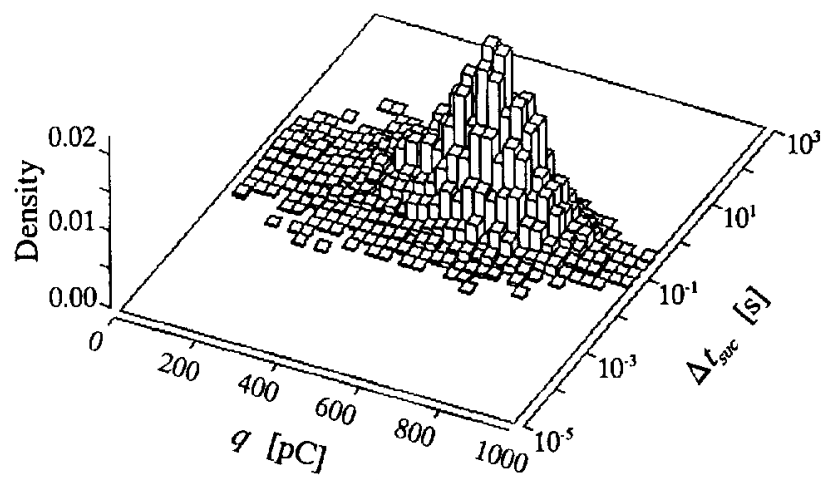

Figure 11

$H\left(q, \Delta t_{\text {suc }}\right)$ histogram for corona in oil at $70 \mathrm{kV}$ (needle radius $35 \mu \mathrm{m}$ ).

stated that for ac, most of the known characteristic parameters can be derived from the 3-dimensional discharge magnitude-phase histogram $H(q, \varphi)$, see Figure 9.

\section{2. de DISCHARGE DIAGNOSTICS}

At $\mathrm{dc}$, no approach to classify and recognize discharge defects is known to the author. The basic quantities which can be measured for each single discharge are the discharge magnitude $q_{i}$ and the time of occurrence $t_{i}$.

If for ac all known classification procedures can be based on the 3-dimensional discharge magnitude-phase histogram $H(q, \varphi)$, then the classification for dc should be based on a 3-dimensional distribution as well. This distribution must contain the discharge magnitude as one parameter and an additional time parameter (similar to the phase $\varphi$ has been used for ac). The author suggests the use of the $H\left(q, \Delta t_{s u c}\right)$ histogram as a base for classification and recognition procedures at dc voltage (see Figure 10). Because of the mathematical similarity of $H(q, \varphi)$ and $H\left(q, \Delta t_{s u c}\right)$, all knowledge gained for ac discharge pattern recognition (based on $H(q, \varphi)$ ) can be applied for the recognition of PD pattern at dc voltage based on $H\left(q, \Delta t_{s u c}\right)$.

In [22] the 3-dimensional $H\left(q, \Delta t_{s u c}\right)$ histogram has been found to be characteristic for specific types of discharges (see Figures 11 to 13 ). That means that dc discharge classification and recognition should be possible. Based on the $H\left(q, \Delta t_{s u c}\right)$ histograms an expert could visibly recognize some defects. For instance, for corona in air a very narrow peak is characteristic (Figure 12). For internal discharges (Figures 13) the maximum density is registered for the smallest measured discharges.

In order to automate, the classification must be based on a set of characteristic parameters. Automated classification is made by comparing the characteristic parameters of known defects with those of unknown defects. Similar to the ac case, there are the following possibilities.

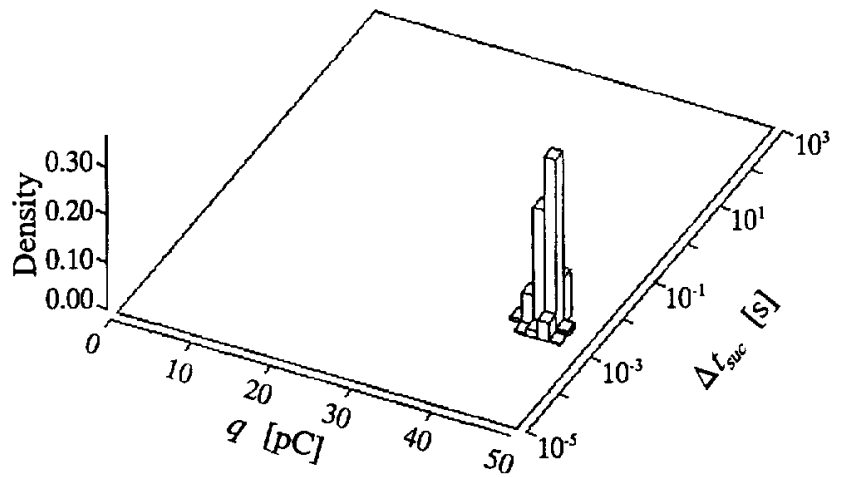

Figure 12.

$H\left(q, \Delta t_{\text {suc }}\right)$ histogram for corona in air at $8 \mathrm{kV}$ (needle radius $40 \mu \mathrm{m}$ ).

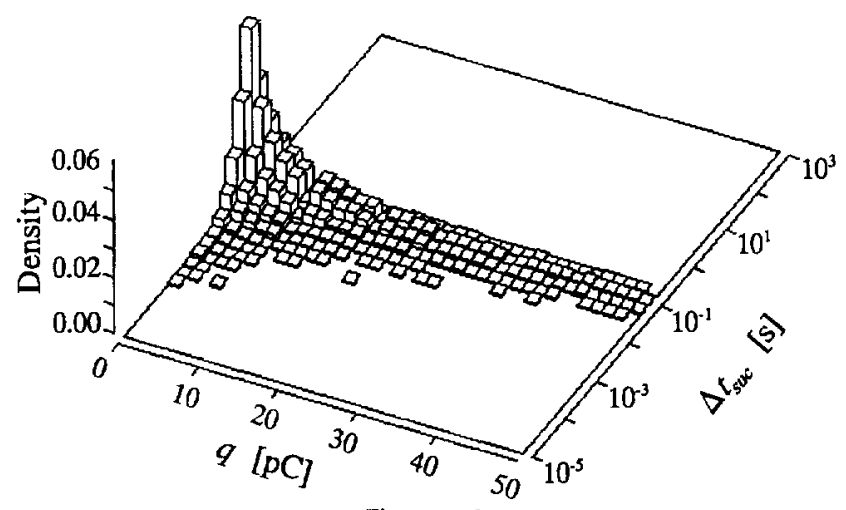

Figure 13.

$H\left(q, \Delta t_{s u c}\right)$ histogram for internal discharges in polyethylene (sample height $0.6 \mathrm{~mm}$, void diameter $10 \mathrm{~mm}$, void height $0.2 \mathrm{~mm}$ ).

The 3-dimensional $H\left(q, \Delta t_{s u c}\right)$ histogram could be used as a set of characteristic parameters and stored in a database. For the classification neural networks could be used as is reported for ac in $[35,36]$. Fractal features could be used as characteristic parameters to describe the surface of the 3-dimensional $H\left(q, \Delta t_{s u c}\right)$ histogram as reported in [21].

Some 2-dimensional histograms such as discharge magnitude histogram $H(q)$ or the time separation histogram $H\left(\Delta t_{s u c}\right)$ can be derived from the $H\left(q, \Delta t_{s u c}\right)$ histogram. Skewness $S_{k}$ and kurtosis $K_{u}$ could be used as characteristic parameters to describe the shape of these 2dimensional histograms as reported for ac in [27-29]. The differences in the 3-dimensional histogram (Figures 11 to 13) lead to differences in the derived 2-dimensional histograms as well (Figures 14 to 16). The classification and recognition should not just be based on a single 2dimensional histogram. For instance, the values of $K_{u}$ and $S_{k}$ of the magnitude histogram for corona in air (Figure 14(a)) and corona in oil (Figure 15(a)) do not differ very much. But there are clear differences of the $S_{k}$ and $K_{u}$ values for the time separation histogram of both de- 
fects Figures 14 (b) and 15 (b). The presence of a ripple on the dc test voltage can affect the distribution of the time separation [2] (Figure 16) which can influence the result of recognition.
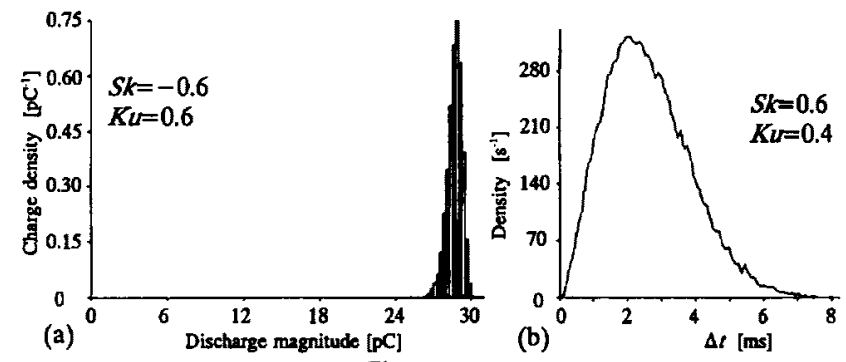

Figure 14.

(a) The discharge magnitude histogram $H(q)$ and (b) the time separation histogram $H(\Delta t)$ for corona in air $\left(V_{0}=7 \mathrm{kV}\right)$.

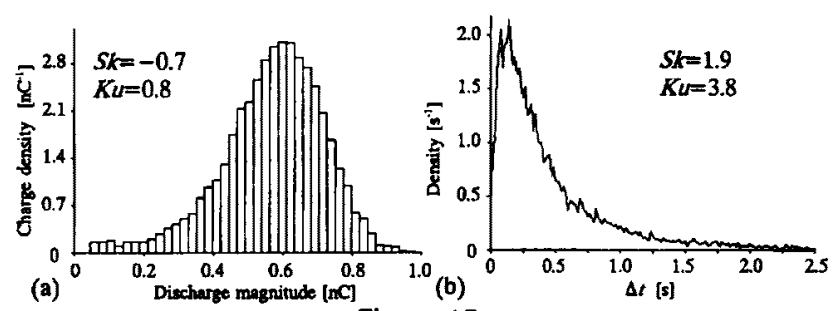

Figure 15.

(a) The discharge magnitude histogram (b) and the time separation histogram $H(\Delta t)$ for corona in oil $\left(V_{0}=70 \mathrm{kV}\right)$.

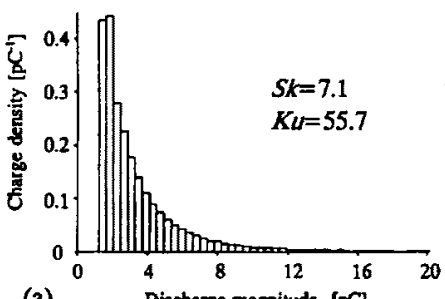

(a)

Figure 16

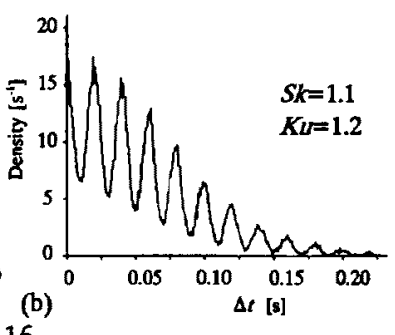

(a) Histogram of the discharge magnitude (b) and of the discharge separations for internal discharges $\left(h_{S}=0.3 \mathrm{~mm}, h_{V}=0.1 \mathrm{~mm}, d_{V}=6\right.$ $\mathrm{mm}, V_{0}=20 \mathrm{kV}$ ). The oscillation in (b) is caused by an ac ripple on the de test voltage [2].

\section{CONCLUSIONS}

1. An overview on de PD is given.

2. The time lag/recovery model was developed to describe the discharge behavior of defects with a single discharge site. It can be used to study physical discharge properties based on statistical measurements. It has been shown to be applicable for corona discharges in air and for internal partial discharges.

3. The classification of partial discharges at dc is suggested to be based on the 3-dimensional histogram
$H\left(q, \Delta t_{s u c}\right)$. All classification methods developed for ac may be used at dc as well if the classification is based on the $H\left(q, \Delta t_{s u c}\right)$ distribution.

\section{REFERENCES}

[1] J. C. Devins, "The Physics of Partial Discharges in Solid Dielectrics" IEEE Trans. on El. Ins., Vol. 19, No. 5, pp. 475-495, 1984.

[2] F. H. Kreuger, and U. Fromm, "Partial Discharges in Gaseous Voids for dc Voltage", Japan J. Appl. Phys. Vol. 33, Part 1, No. 2, pp. 1079-1084, February 1994.

[3] K. Nakamura, and Y. Kamijo, "Fluctuation Mechanism of dc Partial Discharge in Polyethylene and Impulse Noise in Submarina Cables", Japan J. Appl. Phys. Vol. 15 Part 1, No. 5, pp. 865-870, 1976.

[4] B. Salvage, and N. R. Steinberg, "Discharge Repetition in an Air-filled Cavity in a Solid Dielectric under Direct-voltage Conditions", Electronics Letters, Vol. 2, No. 11, pp. 432-433, 1966.

[5] S. Shihab, Teilentladungen in Hohlräumen von Polymeren Isolierstoffen Bei Hoher Gleichspannung, Diss. TU Braunschweig, Germany, 1972.

[6] K. Watanabe, "Dc Partial Discharges and Current in Polyethylene", Japan J. Appl. Phys. Vol. 17, Part 1, No. 3, pp. 483-490, March 1978.

[7] R. J. Van Brunt, M. Misakian, S. V. Kulkarni, V. K. Lakdawala, "Influence of a Dielectric Barrier on the Statistic Behavior of Trichel-pulse Corona", IEEE Trans. EI, Vol. 26, No. 3, pp. 405-415, 1991.

[8] R. S. Bever, "Ramp Technique for dc Partial Discharge Testing", IEEE Trans. EI, Vol. 20, No. 1, pp. 38-45, 1985.

[9] B. Salvage, and W. Sam, "Detection and Measurement of Discharges in Solid Insulation under Direct-voltage Conditions", Proc. IEE, Vol. 114, No. 9, pp. 1334-1336, 1967.

[10] E. Takahashi, Y. Tsutsumi, K. Okuyama, and F. Ogata, "Partial Discharge Characteristics of Oilimmersed Insulation Systems under dc, Combined ac-dc and dc Reversed Polarity Voltage", IEEE Trans. on PAS, Vol. 95, No. 1, pp. 411-421, 1976.

[11] IEEE Working Group on Dielectric Tests for HVDC Stressed Transformers and Reactors, "Recomended Dielectric Test Procedures for Converter Transformers and Smoothingreactors", IEEE Trans. on PWRD, Vol. 1, No. 3, pp. 161-166, 1986.

[12] G. S. Kutschinski, "Ermittlung Der Lebensdauer Der Öpapierisolierung von Kondensatoren Aus Den Kennwerten Der Teilentladungen", Elektrie, Vol. 22. pp. 183-186, 1968. 
[13] R. J. Van Brunt, "Stochastic Properties of Partialdischarge Phenornena", IEEE Trans. EI, Vol. 26, No. 5, pp. 902-948, 1991.

[14] E. C. Rogers, and D. J. Skipper, "Gaseous Discharge Phenomena in HV dc Cable Dielectrics", Proc. IEE, Vol. 107, Part A, pp. 241-254, 1960.

[15] T. Ficker, and J. Ikula, "Spark and Glow dc-partial Discharges in Dielectrics", Japan J. Appl. Phys. Vol. 23, No. 9, pp. 1263-1264, 1984.

[16] U. F. Fromm, and P. H. F. Morshuis, "The Discharge Mechanism in Gaseous Voids at dc Voltage", Submitted to 9th ISH, Paper 4154, Graz, 28 Aug.-1 Sep. 1995.

[17] K. B. Müller, Über Das Verhalten Extrudierter Pe-kabel Bei Hoher Gleichspannungs- Langzeitbelastung, Diss. TH Darmstadt, Germany, 1976.

[18] N. H. Malik, and A. A. Alrainy, "Statistical Variation of dc Corona Pulse Amplitudes in Point-toplane Gaps", IEEE Trans. on EI, Vol. 22, No. 6, pp. 825-829, 1987.

[19] J. Macur, K. Domansky, and J. Sikula, "Stochastic Character of Partial Discharges in Insulators", J. Appl. Phys., Vol. 67, No. 1, pp. 540-542, 1990.

[20] R. J. Van Brunt, K. L. Stricklett, J. P. Steiner, S. V. Kulkarni, "Recent Advances in Partial Discharge Measurement Capabilities at the National Institute of Standards and Technology", IEEE Trans. on EI, Vol. 27, No. 1, pp. 114-129, 1992.

[21] U. Fromm, and P. H. F. Morshuis, "Partial Discharge Classification at dc Voltage", 5 th ICSD, Leicester, paper 7.1.9, 10-13 July 1995.

[22] U. Fromm, Partial Discharge and Breakdown Testing at High dc Voltage, Diss. TU Delft (The Netherlands), Delft University Press, ISBN 90407-1155-0, 1995.

[23] J. C. J. Eeman, "Experimental Partial Discharge Detection Equipment for dc and dc Ramp HV Testing", Proc. of the Europ. Space Power Conf., ESA SP-320, Florence 2-6 Sep., August 1991.

[24] U. Fromm, and F. H. Kreuger, "Statistical Behavior of Partial Discharges at dc Voltage", Japan J. Appl. Phys. Vol. 33, Part 1, No. 12A, pp. 67086715, 1994.

[25] P. H. F. Morshuis, Partial Discharge Mechanisms, Diss. TU Delft (The Netherlands), Delft University Press, ISBN 90-6275-931-9, 1993.
[26] "Recognition of Discharges", CIGRE, Electra, Vol. 11, pp. 61-98, 1969.

[27] E. Gulski, Computer-aided Recognition of Partial Discharges Using Statistical Tools, Diss. TU Delft (The Netherlands), Delft University Press, ISBN 90-6275-728-6, 1991.

[28] E. Gulski, and A. Krivda, "Neural Networks as a Tool for Recognition of Partial Discharges", IEEE Trans. on Electrical Insulation, Vol. 28, pp. 9841001, 1993. Errata in IEEE Trans. on Dielectrics and Electrical Insulation, Vol. 1, p. 351, 1994.

[29] T. Okamoto, and T. Tanaka, "Novel Partial Discharge Measurement Computer-aided Measurement Systems", IEEE Trans. on Electrical Insulation, Vol. 21, pp. 1015-1019, 1986.

[30] F. H. Kreuger, E. Gulski, and A. Krivda, "Classification of Partial Discharges", IEEE Trans. on Electrical Insulation, Vol. 28, pp. 917-931, 1993.

[31] R. H. Lindeman, P. F. Merenda, and R. Z. Gold, Introduction to Bivariate and Multivariate Analysis, pp. 203-204, Scott, Foresman and Company, 1980.

[32] L. Satish, "Can Fractal Features be used for Recognizing 3-D Partial Discharge Patterns?", IEEE Trans. on DEI, 1995.

[33] S. S. Chen, and J. M. Keller, "On the Calculation of Fractal Features from Images", IEEE Trans. on Pattern Analysis and Machine Intelligence, Vol. 15, No. 10, pp. 1087-1090, 1993.

[34] J. M. Keller, and S. S. Chen, "Texture Description and Segmentation through Fractal Geometry", Computer Vision, Graphics, and Image Processing, Vol. 45, pp. 150-166, 1989.

[35] N. Hozumi, T. Okamoto, and T. Imajo, "Discrimination of Partial Discharge Patterns Using a Neural Network", IEEE Trans. on Electrical Insulation, Vol. 27, pp. 550-556, 1992.

[36] H. Suzuki, and T. Endoh, "Pattern Recognition of Partial Discharge in XLPE Cables Using a Neural Network", IEEE Trans. on Electrical Insulation, Vol. 27, pp. 543-549, 1992.

[37] A. Krivda, Recognition of Discharges. Discrimination and Classification, Diss. TU Delft (The Netherlands), ISBN 90-407-1156-9, Delft University Press, 1995.

Manuscript was received on 19 April 1995, in revised form $13 \mathrm{July}$ 1995. 